\title{
Inhalable nano-embedded microspheres as an emerging way for local treatment of lung carcinoma: Benefits, Methods of preparation \& characterizaton
}

\author{
Dina M. Gaber ', Noha Nafee ${ }^{2,3} \&$ Osama Y. Abdallah ${ }^{2}$ \begin{abstract}
science, Technology and Maritime Transport, Alexandria, Egypt
2 Department of Pharmaceutics, Faculty of Pharmacy, Alexandria University, Alexandria 21521, Egypt

${ }^{3}$ Department of Pharmaceutics, Faculty of Pharmacy, Kuwait University, 13110 Kuwait

Email: Dinagaber@aast.edu,n.nafe3@gmail.com, ossama.y.abdallah@gmail.com
\end{abstract} \\ ' Department of Pharmaceutical Sciences (Pharmaceutics), College of Pharmacy, Arab Academy for
}

Received on, 28 October 2021 - Accepted on, 1 December 2021 - Published on, 16 December 2021

\section{Abstract:}

Lung cancer is the leading cause of cancer deaths worldwide, and this makes it an attractive disease to review and possibly improve therapeutic treatment options. The extreme lethality of lung cancer is ascribed to the lack of early diagnostic strategies as in almost $50 \%$ of the cases the disease is confirmed in stage IV, leaving low chance of survival. The inaccessibility to the deeper portions of the lung for conventional therapy further adds up to the complication in the treatment process. Surgery, radiation, chemotherapy, targeted treatments, and immunotherapy separate or in combination are commonly used to treat lung cancer. However, these treatment types may cause different side effects, and chemotherapy-based regimens appear to have reached a therapeutic plateau. Hence, effective, better-tolerated treatments are needed to address and hopefully overcome this conundrum. Nanocarriers through inhalational route offer many advantages like; 1) they achieve uniform distribution of drug among the alveoli, 2) better solubilization of the drug, 3) sustained drug release which subsequently decreases dosing frequency, 4) better patient compliance, 5) lesser side effects, and 6) improved drug internalization to the cells. Therefore, targeted inhalable NP delivery to the lungs is a potential area of research in cancer nanotechnology that catches the attention of many formulation scientists, oncologists, and biomedical researchers. Based on this literature review, we will discuss the development, characterization, and benefits of inhalable nanocarriers for local treatment of lung carcinoma.

Key words: Nanocarriers- lung carcinomamicroparticles

\section{INTRODUCTION}

\subsection{Incidence and etiology of lung cancer}

Lung cancer now accounts for $23 \%$ of all cancer-related deaths globally, outnumbering the combined deaths from breast, colon, and prostate cancer ${ }^{[1,2]}$. It can start in the cells lining the bronchi and parts of the lung such as the bronchioles or alveoli. Further, it is thought to start as areas of pre-cancerous changes in the lung.

\subsection{Different categories of lung cancer}

Lung cancer is mainly categorized into two forms; namely non-small-cell lung carcinoma (NSCLC) (the most frequent kind of lung cancer, accounting for 85 percent of cases) and small-cell lung carcinoma $(\mathrm{SCLC})^{[3]}$. NSCLC is further categorized as epidermoid, large cell, bronchoalveolar, adenocarcinoma, and squamous cell carcinoma ${ }^{[2]}$. These NSCLC forms are histologically distinct from each other. It responds to the chemotherapy; but, their response varies according to treatment protocol. On the other hand, SCLC rarely occurs but shows fast metastasis and aggressive growth, with average survival of merely 4 months if not treated ${ }^{[4]}$.

\subsection{Conventional ways for lung cancer treatment}

Till date, chemotherapy, radiotherapy, and/or invasive surgical procedures have been the most common modalities of cancer treatment. Such conventional therapy, does not distinguish between cancer and healthy cells, resulting in significant side effects as well as a poor tumour response to treatment due to nonspecific bioavailability of the administered anticancer agent. Thus, the lack of efficacy of intravenous chemotherapy against lung tumors results from a combination of (usually) late diagnosis, and inadequate drug access to lung tissue following intravenous administration [5]. Despite the introduction of targeted and tailored therapies to patients according to individual biological tumor characteristics, the overall survival rates have failed to meet the expected progression-free survival or overall survival ${ }^{[6]}$. Moreover, acquired resistance to cytotoxic agents has been observed, mainly involving the apoptotic mechanism in NSCLC cell lines ${ }^{[7]}$. In SCLC, acquired resistance has been observed and fiveyear survival remains less than $10 \%$, despite the use of various drug combinations. Therefore, novel therapies are in great demand.

Thus, the complete eradication of lung cancer requires a new approach such as utility of nano-scale materials. It is by the virtue of nanoscale dimension of lung cancer therapeutic and/or diagnostic system that they are capable of effectively transcending bronchial epithelium 
barrier and accumulating in deep lung regions. Some of such nanoscale formulations that have given promising results include nanogels or nano-sprays which are intratracheally administered into the lungs, and the results have confirmed that intratracheal means of drug delivery for lung cancer therapy are much better than the parenteral route [8]. In one such approach, inhalable deoxycholic acid-modified glycol chitosan (DOCA-GC) nanogels containing palmityl acylated exendin-4 (Ex4-C16) were synthesized for treatment of hyperglycemia.

The therapeutic efficacy of this nanogel formulation was monitored in type 2 diabetic $\mathrm{db} / \mathrm{db}$ mice, and the cytotoxicity associated with them was established by using A549 and Calu-3 cell lines. The use of chitosanbased nanogels for pulmonary delivery did not instigate any immune response and prolonged hyperglycemic effect even at lower concentration of drug. This work established the possibility of using such nanogel-based pulmonary delivery system for delivery of anticancer drugs specifically to lung cancer cells $[9,10]$.

\subsection{Emerging applications of nanoparticles for lung cancer diagnosis and therapy}

Since, nanoparticles (NPs) have attractivecharacteristics like small particle size, large surface area, and the capability of tailoring their surface properties, they therefore have several advantages over other delivery systems ${ }^{[11]}$. Additionally, it has been proven that NPbased drug delivery systems assure passive (size-based targeting due to their size up to $\sim 100 \mathrm{~nm}$ ) as well active targeting (surface functionalization by targeting ligand) and enhanced therapeutic efficacy of anticancer agents [12].

The applications of these nanoparticles in cancer therapies have been effective to a great extent owing to their inherent small dimensions which enable them to specifically accumulate in tumor cells as they permeate through the leaky vasculature in the vicinity of tumor cell mass (enhanced permeability and retention effect, EPR).

The poorly developed lymphatic drainage also contributes indirectly to NP accumulation at the site of the tumor. Another benefit of nanoscale systems is that they may efficiently overcome kidney clearance and thus give adequate blood circulation time for the drugs they transport. In other words, NPs can limit the biodistribution profile of anticancer medications and focus them to tumour areas, increasing therapeutic efficiency and lowering nonspecific toxicity [11].

\section{Local versus systemic treatment of lung carcinoma}

The majority of chemotherapeutics come in intravenous (iv) forms. Furthermore, some important chemotherapeutics used to treat lung malignancies are extremely lipophilic, necessitating greater doses and/ or surfactant-based solubilization to improve systemic drug availability. In addition, oral administration of cancer chemotherapeutics is often limited due to first-pass metabolism [13]. However, systemic drug bioavailability is not the only concern here, as even at higher dose or systemic availability, only limited quantity of drugs is delivered to lung tumor. The majority of chemotherapeutics act on normal tissues due to their non-targeting nature, causing side effects. Nevertheless, drugs already being used for systemic administration have been successfully administered regionally in various types of cancer. The concept of local drug delivery is proposed as a method for delivering high drug concentrations to the target site while preventing exposure of vital organs to toxic drug concentrations in the systemic circulation. In this way, local delivery can play an important role in safer chemotherapy with better patient compliance and minimized systemic side effects. The respiratory system has a large surface area, thin alveolar epithelium, rapid absorption, lack of firstpass metabolism, high bioavailability, and the capacity to absorb large quantities of drug, making it an optimal route of drug administration. In addition, it is considered as a needle-free approach that offer better comfort to the subjects ${ }^{[13]}$.

\section{Targeted inhalable nanoparticle for lung carcinoma}

Pulmonary delivery via inhalation is a common technique of drug administration to patients with a variety of lung diseases. But the airway geometry of the lungs poses a challenge for delivery into the alveoli $[14,15]$. However, delivery of individual nanoparticles to the lungs appears to be a problematic, as due to their small sizes $(<1 \mu \mathrm{m})$ which increased their probability of exhalation before deposition [16]

Therefore, to successfully deliver nanoparticles by inhalation, they first have to be transformed into microscale nanocomposite structures having theoretical aerodynamic diameter between 1 and $5 \mu \mathrm{m}$ [17]. There are three clinically successful pulmonary inhalation pharmaceutical dosage forms based on device classes; namely, nebulizers (Nebs), pressurized metered dose inhalers (PMDIs) and dry powder inhalers (DPIs). DPIs are breath-actuated devices that deliver a dry powder drug through shear-induced aerosolization. They may contain respirable powdered drugs alone or blended with non- respirable carriers.

DPIs offer manyadvantages including highencapsulating ability, long term stability, no coordination of actuation and inhalation, no liquid propellant, an extended release profile, improved tolerability, reduced toxicity, easy to use and non-invasiveness. Based on the mechanisms of particle dispersion and aerosolization, the DPI devices are further categorized as passive or active devices. DPI is a rapidly growing sector of the pulmonary inhalation pharmaceutical market which is evident by the increasing number of successful products in the market. DPIs have two potential problems concerning relatively low fine particle fraction (FPF) and emitted dose (ED) which can be attributed to insufficient particle dispersion by the patient or DPI device, aerosol dispersion inefficiency, or the powder formulation itself ${ }^{[15]}$. 
Over the past decade, a new direction in nanotechnology has been raised to focus on targeting to lung diseases including cancer. The focus is to combine the nanotechnology-based therapeutic delivery with pulmonary/ inhalational route of administration. This strategy has been encouraged due to the possible usefulness of lung as a portal for drug entrance, including peptides and proteins. The lungs are well-organized entrance for drugs to the bloodstream as they have large surface area for absorption $(\sim 100 \mathrm{~m} 2)$, with very thin absorption membrane $(0.1-0.2 \mu \mathrm{m})$. Furthermore, the lungs show comparatively lesser local metabolic activity, and unlike the oral route of drug administration, pulmonary/ inhalation route is not vulnerable to firstpass metabolism [18].

Nanocarriers through inhalational route offer many advantages like; 1) they achieve uniform distribution of drug among the alveoli, 2) better solubilization of the drug, 3) sustained drug release which subsequently decreases dosing frequency, 4) better patient compliance, 5) lesser side effects, and 6) improved drug internalization to the cells ${ }^{[11]}$. Therefore, targeted inhalable NP delivery to the lungs is a potential area of research in cancer nanotechnology that catches the attention of many formulation scientists, oncologists, and biomedical researchers. Here, we discuss the challenges in delivery of chemotherapeutics to lung cancer, the significance of applying inhalable NPs in lungs cancer drug targeting, and the concern of toxicity in using this approach. Nevertheless, to our knowledge there is no approved inhalation product for local treatment of lung cancer yet available in the international market.

\section{Dry powder inhalers and nanoparticulate powders for inhalation}

Nanoparticles are considered to be promising carriers for pulmonary drug delivery. The differences between microparticles and nanoparticles extend beyond just the size, having larger surface area to volume ratios. Nanoparticles can have a higher drug loading capacity, using less polymers, better cross permeability barriers, increased cellular uptake, longer lung retention and in airway nanoparticles have better chances of mucus penetration [14]. This improves dissolution properties where decreasing the particle size increases the solubility and intracellular drug delivery potential.

Studies have also demonstrated that particles with decreased size are better internalized by cells. Microspheres or in another term nanocomposite particles consist of drug-loaded nanoparticles and excipients. Nanoparticles are combined with a matrix to form micro-size particles, the $3 \mu \mathrm{m}$ size of which is the most effective to deposit deeply into the lung site. Upon deposition in the lung and exposure to the humid environment and the lung lining fluid, the matrix dissolves and readily decomposes into primary drugloaded nanoparticles in the surface layer of alveoli as shown in Figure ${ }^{[14]}$.

Nanoparticles can escape mucociliary clearance and recognition of alveolar macrophages. Therefore, after depositing to the surface layer of the alveoli, drugloaded nanoparticles will immigrate near the epithelial cells and be taken up by them. Yang et al. ${ }^{[19]}$, successfully formulated a salmon calcitonin adsorbed polymeric PLGA nanospheres which was coated onto a lactose carrier to form nanocomposites. The nanocomposite particles had efficient lung deposition and a rapid release of salmon calcitonin occurred. Another interesting aspect was explored by Yang et al. ${ }^{[17]}$, who developed DPI formulation of hybrid nanoparticles composed of PLGA and soybean lecithin as the polymer and lipid constituents, respectively. The hybrid nanoparticles are transformed into inhalable micro-scale nanocomposite structures by a novel technique based on electrostatically-driven adsorption of nanoparticles onto the polysaccharide chitosan carrier particles.

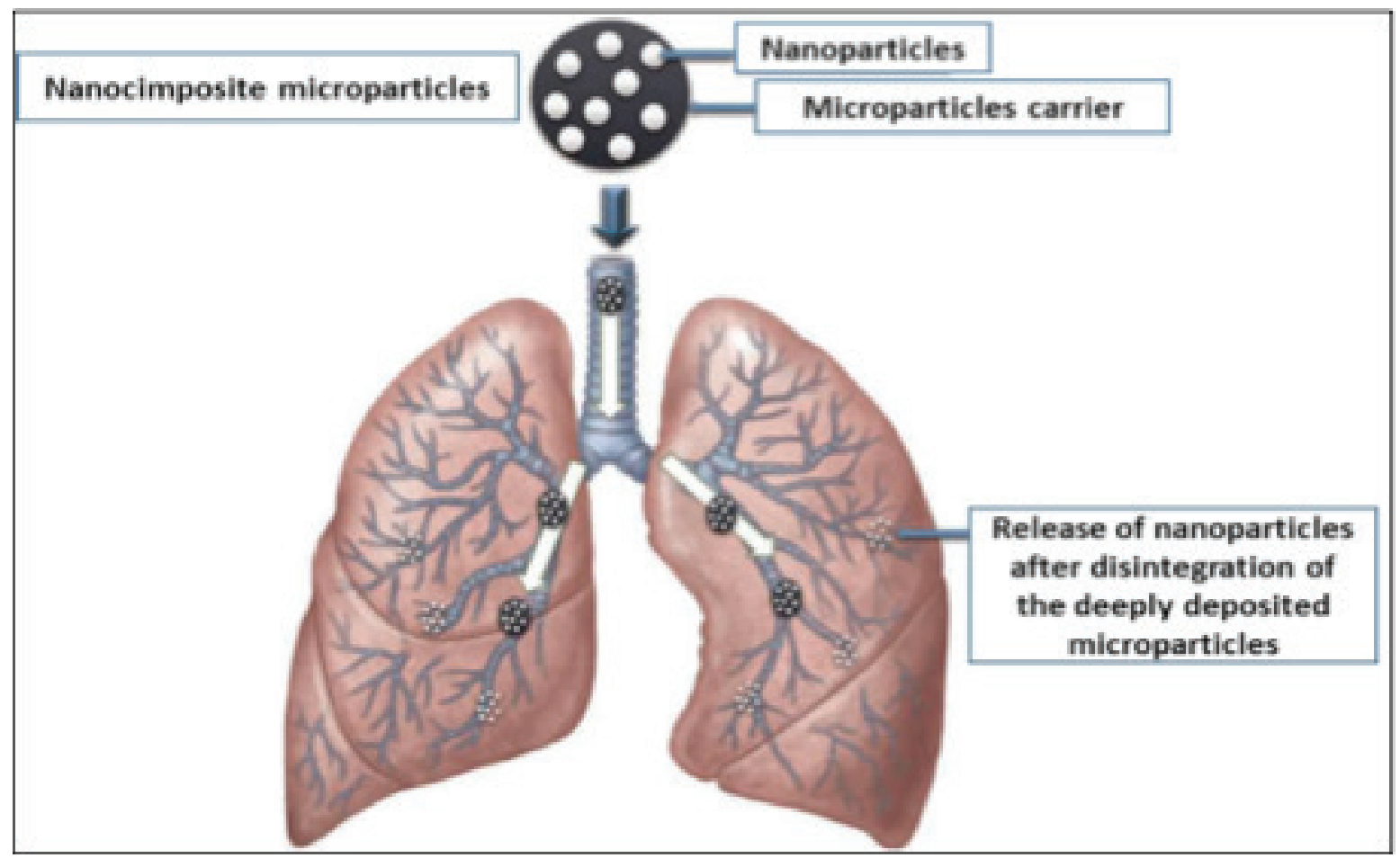

Figure 1: Decomposition of nanocomposite microparticles into primary nanoparticles upon administration [14] . 


\subsection{Engineering of nanocomposite powders for inhalation as DPIs}

Particles with aerodynamic diameters ranging from 1-5 $\mu \mathrm{m}$ are optimal for lung deposition. The complex nature of interparticulate interactions on the low micron-sized scale, together with the extensive application of dry powder formulations and the specific of aerodynamic behavior within the inhaler device and the respiratory system, have hindered the development of such a dosage form. The pharmaceutical industry has adopted a variety of techniques to incorporate desirable characteristics into inhalable multiparticulate systems, including narrow particle-size distribution, improved dispersibility, enhanced drug stability, optimized bioavailability, sustained release and/ or precise targeting [20]. The most sophisticated and advanced manufacturing technologies utilized are:

\subsubsection{Milling process}

Milling is a common pharmaceutical processing procedure for reducing primary particle size and producing a dry powder with the desired particle size range ${ }^{[21]}$. Vibration milling, ball milling and, in particular, jet-milling (fluid energy) are well-established and well validated techniques used to manufacture dry powders for inhalation. A ball mill is essentially a rotating cylinder loaded with a drug and the "milling media" (i.e., balls that grind the drug between each other as they tumble inside the mill). Ball milling is time consuming and poorly scalable process. On the other hand, jet milling is the most widely used method for producing respirable aerosol particles in the solid-state. The basic procedure of jet-milling is to grind a bulk crystallized particles into small particles by one of the following mechanical forces: pressure, friction, attrition, impact, or shear. Particles with diameters down to approximately $1 \mu \mathrm{m}$ can be produced using this technique. Although no approved inhaled drugs have been produced from the milling process to date, the process has been explored with several inhaled drugs, including beclomethasone dipropionate, fluticasone propionate and ciclosonide ${ }^{[22]}$. In a recent study conducted by Onoue et al. ${ }^{[23]}$, tranilast, an antiallergic agent was prepared as a nanocrystal solid dispersion which was freeze dried. The resulting solidstate particles were micronized by gas-jet milling to render them into the respirable size range and blended with large $(50 \mu \mathrm{m})$ non-respirable lactose monohydrate carrier particles. These particles produced dry powder inhalation aerosols with $97.9 \%$ emitted dose (ED) and $59.4 \%$ fine particle fraction (FPF) values. The in vivo characteristics showed a notable anti-inflammatory performance of the formulation.

\subsubsection{Spray drying}

Spray drying is a pharmaceutical manufacturing process used to efficiently produce respirable colloidal particles in the solid-state. In this process, the drug solution or suspension is introduced at a high pressure via an atomizing nozzle with spray-air into a heated chamber where, the solvent evaporates and the solid dries out followed by separation of the particles using a cyclone separator. Compared to milling, spray drying produces more spherical particles. Such particles are characterized by a lower area of contact and smaller, more homogeneous particle-size distribution that results in a higher respirable fraction than mechanically micronized drugs. Nevertheless, particles from spray drying process are not always spherical and may have convoluted surfaces, holes and voids [24]. In fact, the shape is influenced by the drying rate, surface tension and viscosity of the liquid. One of the principal purposes of aerosolizing spray-dried powders is to achieve particle diameters of several micrometers with a narrow particle size distribution. This ensures, assuming an appropriate aerodynamic diameter, a maximum deposition of the embedded drug in the tracheo-bronchial and deep alveoli regions at normal inhalation rates [25]. The principal advantages of spray drying with respect to pulmonary drug delivery are the ability to manipulate and control a variety of parameters such as solvent composition, solute concentration, solution and gas feed rate, temperature and relative humidity and droplet size. This allows optimization of particle characteristics namely; size, size distribution, morphology and density, in addition to macroscopic powder properties such as bulk density, flowability and dispersibility. Moreover, spray dryers are available at different production scales and industrial scale up is easy. However, degradation during the spray drying process may be a problem for some macromolecules as a result of a number of factors such as thermal stress during droplet drying, high shear stress in the nozzle and peptide/ protein adsorption at the greatly expanded liquid/air interface of the spray solution [25].

\subsubsection{Spray-freeze drying}

Spray freeze drying is an advanced particle engineering method which combines spray drying and freeze-drying processing steps. This technique involves the atomization of an aqueous drug solution into a spray chamber filled with a cryogenic liquid (liquid nitrogen) or halocarbon refrigerant such as chlorofluorocarbon or fluorocarbon [26]. Since the normal boiling point for such a liquid is very low, the droplets are quickly frozen. Lyophilizing these frozen droplets results in porous spherical particles suitable for inhalation. Cheow et al. ${ }^{[27]}$, prepared spray freeze dried poly-caprolactone nanoparticles containing levofloxacin. This method produced inhalable particles with low density, smooth surface morphology and good aqueous redispersibility. Furthermore, they proved that polyvinyl alcohol and mannitol as suitable adjuvants can be processed with spray freeze drying. Unlike spray drying, spray freeze drying is conducted at sub ambient temperature, and has, therefore, been used to formulate a significant number of thermolabile and highly potent therapeutic proteins/ peptides into dry powder inhalation products ${ }^{[27]}$. Wang et al. ${ }^{[28]}$, performed comparative studies of employing spray drying and spray freeze drying to produce inhalable dry powder form of levofloxacin-loaded lipid/ polymer hybrid nanoparticles. Results showed the superiority of spray freeze drying over spray drying technique as it produces nano- aggregates of larger aerodynamic diameter resulting in easier physical handling, higher yield and reconstitutibility, better flowability, as well as higher ED and FPF. However, spray freeze drying is not as commonly used as spray drying due to complexity, time consuming and cost involved. 


\subsubsection{Supercritical fluid technology}

Supercritical fluids are known as compressed gases or liquids above their critical pressures and temperatures, which possess several fundamental advantages as solvents or non-solvents for pharmaceutical manufacturing. Carbon dioxide is the most commonly used solvent in this technology. The three main supercritical fluid processes are precipitation from supercritical solutions composed of supercritical fluid and solutions, precipitation from gas saturated solutions and precipitation from saturated solutions using supercritical fluid as antisolvent. All three techniques allow production of inhalable particles with a narrow particle size distribution and less charge which allows them to flow more freely and to be more easily dispersed following discharge from a DPI. Despite its potential, supercritical fluid is still an emerging technology that is not much exploited in DPI products because of complicated and expensive high-pressure equipment, low solubility of many pharmaceutical compounds in $\mathrm{CO}^{2}$ and a lack of experience on scaling-up problems [24].

\subsection{Excipients in Dry Powder Inhalers}

In general, excipients are used to enhance the physical or chemical stability of the active pharmaceutical ingredient, its mechanical and pharmaceutical properties such as dissolution and permeation. In fact, excipients are inactive ingredients that are intentionally added to therapeutic products to improve their delivery or efficacy ${ }^{[18]}$. The FDA favors the use of commercially established excipients as well as "generally recognized as safe" (GRAS) substances. It should be noted that the current excipients approved for respiratory drug delivery are very limited in number (Table 1).

Table 1:

List of accepted or interesting additives for DPI formulations ${ }^{[18]}$. Excipients Description Status

\begin{tabular}{|c|c|c|}
\hline $\begin{array}{l}\text { Sugars } \\
\text { - Lactose } \\
\text { - Glucose } \\
\text { - Mannitol - Trehalose }\end{array}$ & Coarse/fine carrier & $\begin{array}{l}\text { - } \quad \text { Approved and commonly used } \\
\left.\text { - } \quad \text { Approved (Bronchodual }{ }^{\circledR}\right)^{* *} \\
\left.\text { - } \quad \text { Approved (Exubera }{ }^{\circledR}\right)^{* *} \\
\text { - } \quad \text { Promising alternative }\end{array}$ \\
\hline $\begin{array}{l}\text { Hydrophobic additives } \\
\text { - Magnesium stearate }\end{array}$ & $\begin{array}{l}\text { Protection from } \\
\text { moisture }\end{array}$ & - Approved (SkyeProtect) ${ }^{*}$ \\
\hline $\begin{array}{l}\text { Lipids } \\
\text { - DPPC*,DSPC*, DMPC*, } \\
\text { cholesterol }\end{array}$ & $\begin{array}{l}\text { Used in liposomes, } \\
\text { matrix, coating }\end{array}$ & $\begin{array}{l}\text { Biocompatible and biodegradable, very } \\
\text { interesting excipients }\end{array}$ \\
\hline $\begin{array}{l}\text { Amino acids } \\
\text { - Leucine, trileucine }\end{array}$ & $\begin{array}{l}\text { Improved aerosol } \\
\text { efficiency }\end{array}$ & $\begin{array}{l}\text { Endogenous substance but no data on lung } \\
\text { toxicity }\end{array}$ \\
\hline $\begin{array}{l}\text { Surfactants } \\
\text { - Poloxamer • Bile salts }\end{array}$ & $\begin{array}{l}\text { Production of light and } \\
\text { porous particles }\end{array}$ & $\begin{array}{l}\text { - May not be pro-inflammatory at low } \\
\text { dose. } \\
\text { - Endogenous substances, may be } \\
\text { accepted but at low dose }(2-5 \%, w / w) \text {. }\end{array}$ \\
\hline $\begin{array}{l}\text { Absorption enhancers } \\
\text { - Chitosan, trimethylchitosan } \\
\text { - Hydroxypropylated- } \beta-C D^{*} \text {, } \\
\text { natural- } \boldsymbol{\gamma - C D}\end{array}$ & $\begin{array}{l}\text { Absorption for proteins } \\
\text { \& peptides }\end{array}$ & $\begin{array}{l}\text { - Pro-inflammatory effect observed } \\
\text { - Promising results }\end{array}$ \\
\hline $\begin{array}{l}\text { Biodegradable polymers } \\
\text { - PLGA* }\end{array}$ & $\begin{array}{l}\text { Used in sustained } \\
\text { release formulations }\end{array}$ & - Immunogenicity effect observed \\
\hline
\end{tabular}

*DPPC; Dipalmitoyl phosphatidyl-choline, DSPC; Distearoyl phosphatidyl-choline DMPC; Dimyristoyl phosphatidylcholine, CD; Cyclodextrin, PLGA; Poly-lactic-co-glycolic acid.

**Examples of marketed products containing approved excipients. 


\section{Models for studying deposition patterns of inhaled therapeutics}

It is important to study the patterns of deposition of microparticles upon pulmonary administration. Some models are attempted to simulate the lung airway structure and have routinely been used to determine the particle deposition patterns in laboratory setup [29]. Lung cast models have long been used for long time to study various characteristics of the lungs. Lung cast models of human, rat, hamster, monkey and dog have been developed using various materials including rubber, resin, metal, alloy and wax. However, because of the higher degree of complexity and the lack of expertise required for those models, they are not widely used. Impactors, including twin-stage impinger, multistage liquid impinger, Andersen cascade impactor and next generation impactor are the most widely used instruments to measure the size and the deposition patterns of particulate formulations delivered via the pulmonary route. However, impactors are not considered as in vitro simulators of lungs, chiefly, due to the discrepancy in the flow rate when compared with that of a functional lung. It is important to keep in mind that the impactor operates at constant flow rate but human lungs have a varying flow rate originating from the breathing cycle ${ }^{[29]}$.

Nevertheless, impactors comprised of different number of stages that are constructed in a pattern such that larger particles having sufficient inertia will impact upon particular stage collection plate as the aerosol stream passes through, whereas relatively smaller particles with insufficient inertia will be carried in the air stream and pass to the next impaction stage ${ }^{[30]}$ (Figure 2).

Thus, impactors can provide useful information regarding particle size on the basis of aerodynamic diameter and can predict the deposition patterns of particulate drug carriers in the respiratory tract. Impactors are useful in predicting in vivo deposition of particles, and hence are important tools in the development and quality control of new pharmaceutical products. However, these methods have some limitations as they do not adequately mimic the upper and lower respiratory tracts ${ }^{[31]}$. An approach to resolve this problem could be capturing images of particulate drug carriers in vivo by means of various methodologies such as gamma scintigraphy, single photon emission computed tomography, positron emission tomography, magnetic resonance image and fluorescence imaging ${ }^{[29]}$. Currently used in vivo imaging techniques can measure total lung deposition and oropharyngeal deposition of particles directly by using radionuclides, non- ionizable radiation and fluorescent dyes ${ }^{[32]}$. Deposition patterns can be examined directly by taking images of lungs or by autoradiography after sacrificing and dissection of animal lungs.

Overall, the use of in vivo imaging techniques is beneficial over in vitro techniques as they can provide an actual picture of regional deposition of particles in the lungs. Moreover, these techniques can be used to evaluate both qualitative and quantitative aspects of particle deposition in the lungs and can be a useful means to understand the relation between deposition of drugs in the lungs and their clinical effects. However, these techniques have some drawbacks including high cost, higher radiation doses, safety hazards, and specialized training required in handling of radio- labelled isotopes.

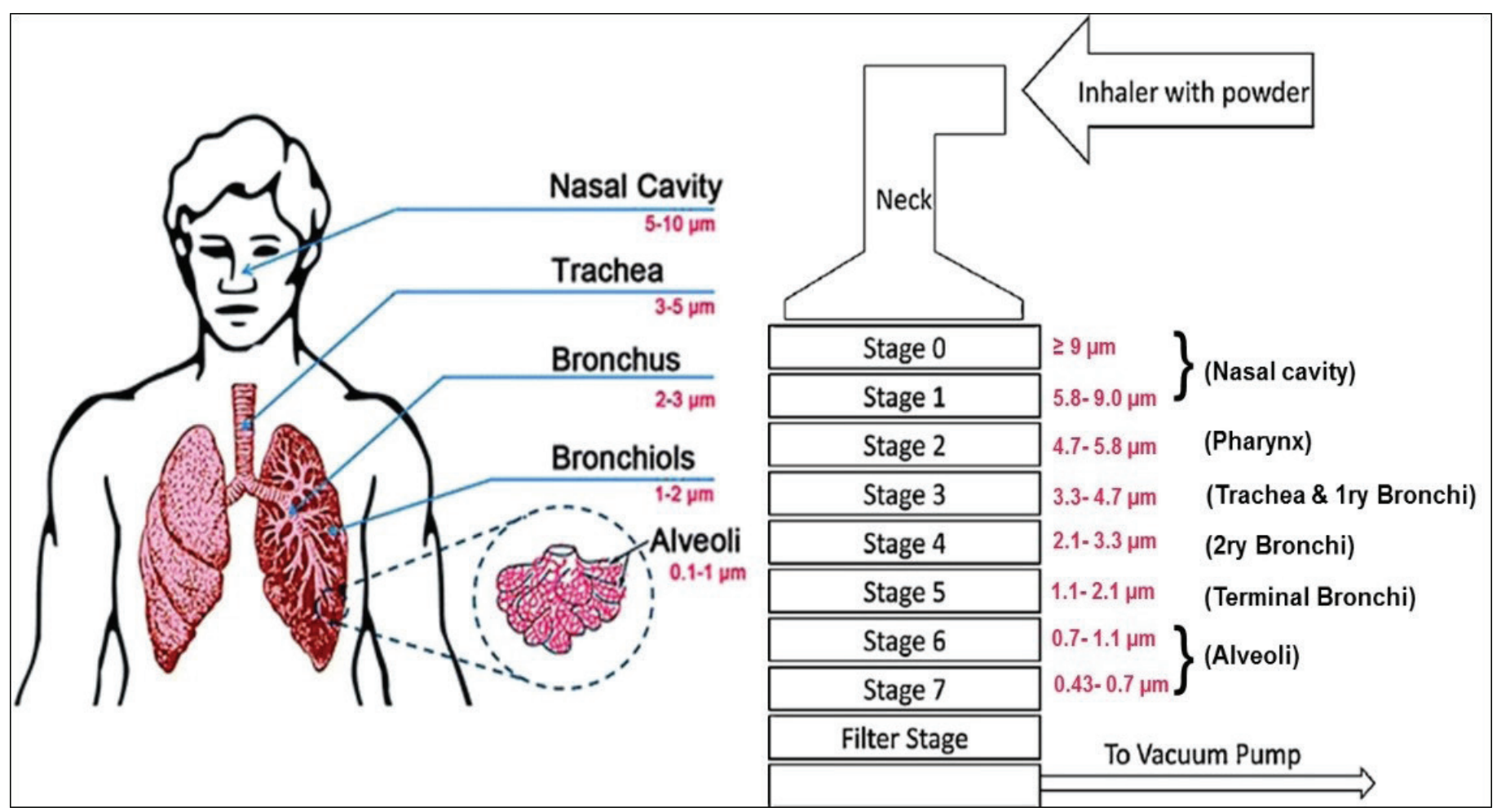




\section{Models to study drug absorption following pulmonary administration}

The absorption profiles of drugs administered via the pulmonary route are evaluated for both locally and systematically acting drugs. For locally acting drugs, pulmonary absorption profiles are determined to assess the amount of drug that is likely to enter the systemic circulation. Pulmonary absorption profiles also give information regarding the amount of drug that would be available locally to produce therapeutic effect in the lungs and overall systemic exposure of the drug.

\subsection{In vitro cell culture models}

In vitro cell culture models have been used extensively to study the uptake, transport and metabolism of drugs by the lungs as they mimic microenvironment of the tissue [15]. The cell lines used include A549, HBE140 and the Calu-3 line. A549 cell line represents the alveolar type II pulmonary epithelial cell, and has been reported to be an ideal model to study the metabolic and macromolecular mechanisms of drug delivery at the alveolar pulmonary epithelium due to the endocytic ability of the pulmonary epithelium and localization of cytochrome P450 systems. Calu-3 and HBE140 model the upper airways (bronchi) which have been utilized extensively in the literatures. For example, the permeability data of small lipophilic molecule (e.g. testosterone) and high molecular weight substance (e.g. fluorescein isothiocyanate-transferrin) across Calu-3 cell line has been examined [33]. The authors demonstrated that this cell line is useful for studying the contributions of bronchial epithelial cells to the mechanism of drug delivery at the respiratory epithelium. Similar conclusions were drawn when the apparent permeability of the glucocorticosteroid budesonide and fluticasone propionate was investigated [34]. However, the main drawbacks of cell culture models are time- consuming isolation and cultivation as well as limited cell lifespan.

\subsection{Ex vivo tissue models}

Ex vivo tissue models are isolated perfused tissue models, which allow studying the mechanism of drug transport, deposition and efficacy in the isolated organ while maintaining its structural and functional integrity [8]. Isolated perfused tissue models provide a more realistic correlation with the in vivo studies compared to the single cell monolayer models. They have been developed for rodents such as rats and rabbits, while attempts have also been made to establish human lung perfusion models. The isolated perfused rat lung model has been used for studying absorption and deposition of inhaled pharmaceutical formulations (Figure 3 ).

This method involves surgical removal of the lung and placing it in an artificial thoracic chamber supplied with atmospheric air and perfusion media. The surgical procedure for isolation of the lungs is fairly complex and requires an extra caution to prevent any tissue damage. Following surgery, drug or formulations can be administered either in perfusate or directly into the lungs using suitable delivery devices in order to evaluate various pharmacokinetic parameters without the influence of the whole body. Following drug administration, continuous sampling is possible from the perfusate outlet from the lungs. This method allows regulation of lung volume, ventilation rates and respiratory patterns ${ }^{[35]}$. However, there are several limitations associated with isolated perfused lung models. One of the main concerns is the viability of tissues over a period of time. Keeping the lungs viable for more than $2-3 \mathrm{~h}$ at $37^{\circ} \mathrm{C}$ has been quite challenging, chiefly due to edema formation.

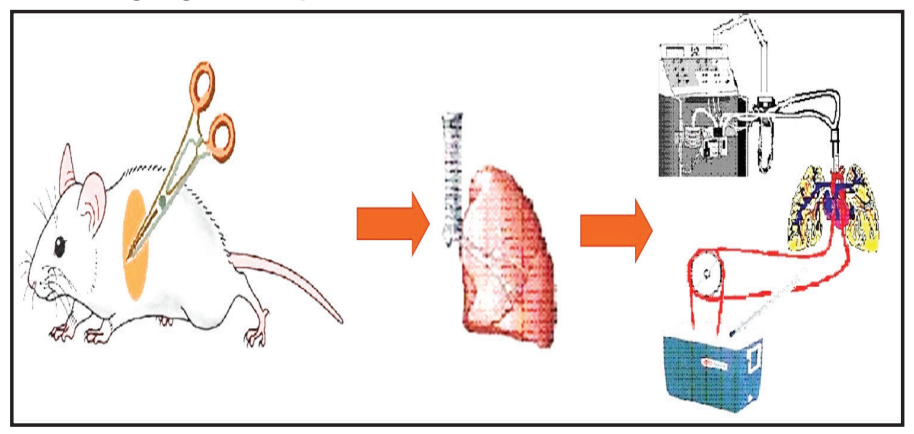

Figure 3: Steps of Ex vivo study on isolated perfused lung models in rats.

\subsection{In vivo animal models}

In vivo intact animal models are mainly used for studying the absorption, distribution and pharmacodynamics of inhaled pharmaceuticals. In earlier methods, the drug was administered to the lungs using an intratracheal tube after surgical exposure of the trachea (Figure 4). However, the proposed method required destructive tissue sampling for each time point which was considered as major limitations for studying pharmacokinetics in intact animals [36]. Recently, this method has largely been replaced by a non-invasive method that uses aerosolizers or insufflators for small animals. For administration of drugs using these devices, the trachea of anesthetized animals is visualized by using a small animal laryngoscope for inserting dry powder insufflator inside the trachea. Drug is spray-instilled into the lungs by pushing the syringe plunger of the device. These devices can deliver liquid or powder in the form of aerosolized droplets or particles to ensure deep lung deposition [37].

In this method, blood sampling is performed by means of surgical catheterization or tail vein without sacrificing animals. However, non-invasive intratracheal administration suffers from a number of limitations. This method does not reflect normal physiological conditions because drugs are administered to anesthetized animals. Moreover, as this method uses anesthetized animals, multiple dosing is not recommended and repeated insertion of inhalation device may cause injury of the trachea. Limitations of direct administration of drugs to anesthetized animals can be overcome by using passive administration chambers such as whole body, nose only and head only exposure chambers. These chambers allow administration of drugs directly to conscious animals thus mimicking a more physiological condition [38]. The difference between 3 models to study drug absorption in vivo is shown in Figure 5. 

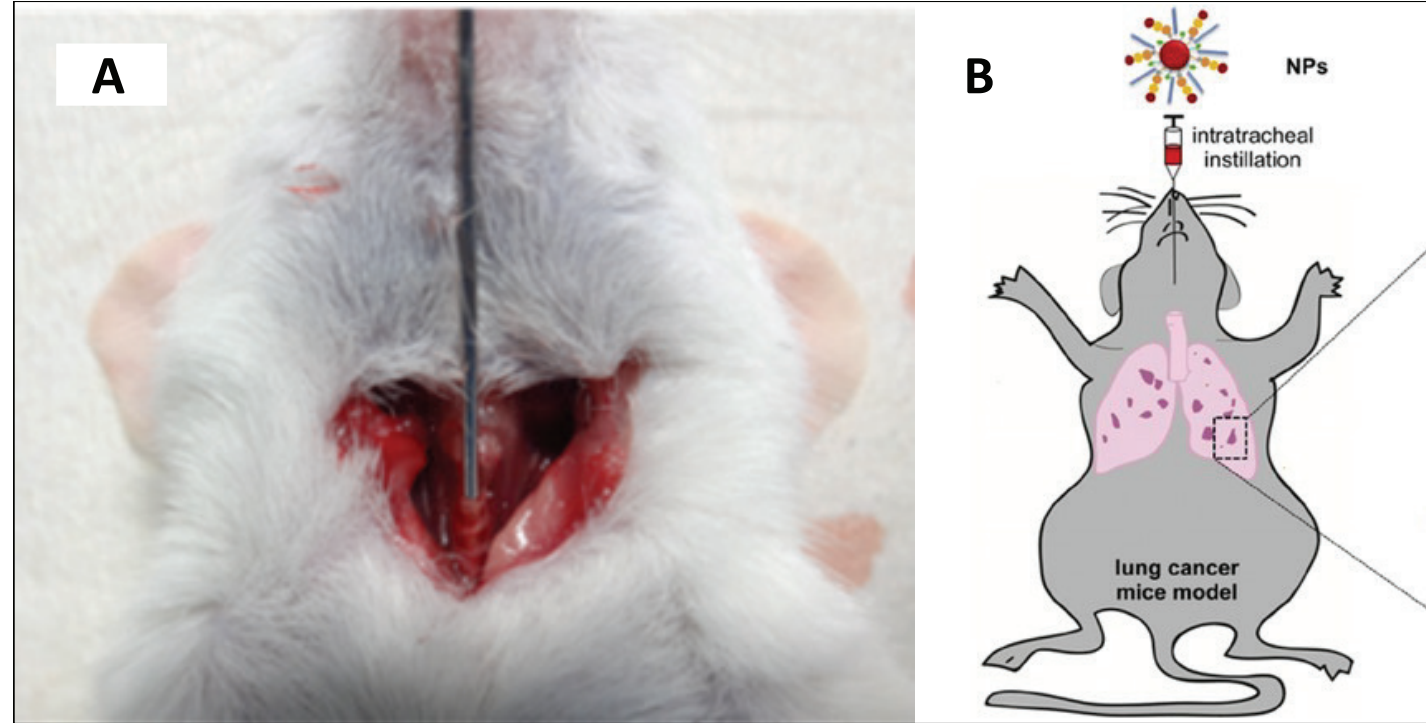

Figure 4: Intratracheal instillation of NPS for local treatment of lung cancer in mice model [39].

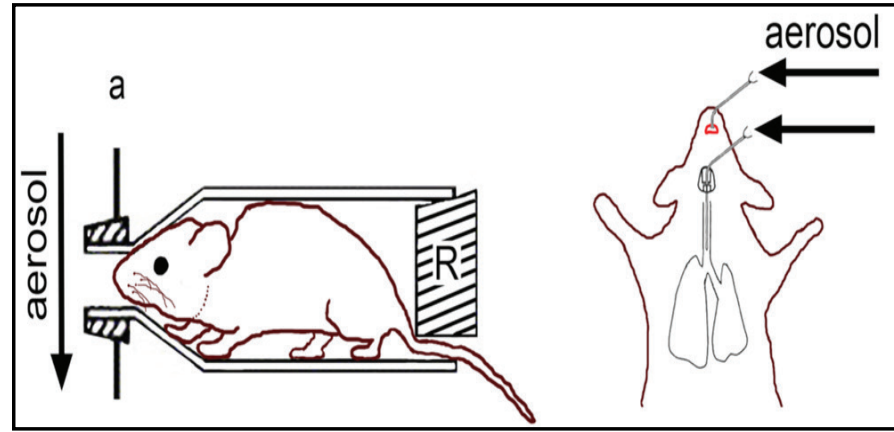

Figure 5: Exposure of rodents to aerosols with 3 different models. A) Intratracheal instillation, B) Oropharyngeal aspiration using insufflators and C) Passive administration chamber with noseonly exposure ${ }^{[40]}$.

\section{Conclusion:}

Cancer is a leading cause of death worldwide. Lung female breast, colorectal and stomach cancers accounted for more than $40 \%$ of cancer cases diagnosed worldwide; with the World Health Organization reporting an estimated 14.1 million new cancer cases worldwide in 2012. Among them, lung cancer is one of the most common, with $16.7 \%$ of all new cases diagnosed in men. Lung cancer is the most common cause of cancerrelated death for men and women and the financial burden to the healthcare system is estimated at $>100$ million dollars annually in Australia. Notably, lung cancer has the highest mortality rate of all common cancers and a miserable dismal rate of less than 5 years. Out of the 8.2 million deaths caused by cancer in 2011 globally, mortality from lung cancers contributed the highest, with 1.3 million deaths alone.

Surgery, chemotherapy and radiation are standard treatment options for lung cancer depending on the stage of malignancy, resectability and overall performance. Chemotherapy is a first-line treatment for advanced stage of lung cancer in which chemotherapeutic drugs are usually administered intravenously for systemic circulation. The use of chemotherapeutic drug is based on the principle of toxic compounds to inhibit the proliferation of cells growing at an abnormal rate. However, it should be noted that the majority of chemotherapeutic drugs is associated with side effects such as pain, nerve damage and skin allergic reactions. Therefore, minimizing the side effects of chemotherapy drugs remains a challenge in the field of cancer chemotherapy. Lung offers numerous advantages as a delivery route for noninvasive drugs for localized therapy of lung cancer. Compared to other delivery methods such as oral or intravenous injection, it is envisaged that the bioavailability of drugs in lung could be enhanced using pulmonary delivery since lung possesses limited intracellular and extracellular drug metabolizing enzyme activities unlike gastrointestinal tract and liver. On top of that, this option also reduces non-reversible tissue damage caused by drugs' cytotoxicity. In addition, higher absorption rate, reduced drug doses and rapid onset of action are among the advantages of pulmonary administration.

\section{References:}

[01] Conway EM, Pikor LA, Kung SH, Hamilton MJ, Lam S, Lam WL, Bennewith KL. "Macrophages, inflammation, and lung cancer." American journal of respiratory and critical care medicine. vol. 193, issue, 2, pp. 116-130, Jan 2016.

[02] Ramalingam SS, Owonikoko TK, Khuri FR. "Lung cancer: New biological insights and recent therapeutic advances." CA: A Cancer Journal for Clinicians, vol. 61, issue 2, pp. 91-112, Feb 2011.

[03] Jemal A, Bray F, Center MM, Ferlay J, Ward E, Forman D. "Global cancer statistics." CA: A Cancer Journal for Clinicians, vol. 6l, issue 2, pp. 69-90, Feb 2011.

[04] Ahmad J, Akhter S, Rizwanullah M, Amin S, Rahman M, Ahmad $M Z$, et al. "Nanotechnology-based inhalation treatments for lung cancer: State of the art." Nanotechnology, Science and Applications, vol. 8, pp. 55-66, Nov 2015.

[05] Wauthoz N, Rosière $R$, Amighi K. "Inhaled cytotoxic chemotherapy: Clinical challenges, recent developments, and future prospects." Expert opinion on drug delivery, vol. 18 , issue 3, pp. 333-354, Mar 2021.

[06] Kang J-H, Song $\mathrm{K}-\mathrm{H}$, Jeong $\mathrm{K}-\mathrm{C}$, Kim S, Choi C, Lee $\mathrm{CH}$, et al. "Involvement of Cox-2 in the metastatic potential of 
chemotherapy-resistant breast cancer cells." BMC cancer, vol. 11, issue 1, pp. 1-13, Dec 2011.

[07] Otterson GA, Villalona-Calero MA, Sharma S, Kris MG, Imondi $A$, Gerber $M$, et al. "Phase I study of inhaled Doxorubicin for patients with metastatic tumors to the lungs." Clinical Cancer Research, vol. 13, issue 4, pp. 1246-1252, Feb 2007.

[08] Sukumar UK, Bhushan B, Dubey P, Matai I, Sachdev A, Packirisamy G. "Emerging applications of nanoparticles for lung cancer diagnosis and therapy." International Nano Letters, vol. 3, issue 1, pp. 1-7, Dec 2013.

[09] Lee J, Lee C, Kim TH, Lee ES, Shin BS, Chi S-C, et al. “Selfassembled glycol chitosan nanogels containing palmitylacylated exendin-4 peptide as a long-acting anti-diabetic inhalation system." Journal of Controlled Release, vol. 161, issue 3, pp. 728-734, Aug 2012.

[10] Paranjpe M, Muller-Goymann CC. “Nanoparticle-mediated pulmonary drug delivery: A review." International Journal of Molecular Sciences, vol. 15, issue 4, pp. 5852-5873, Apr 2014.

[11] Mottaghitalab F, Farokhi M, Fatahi Y, Atyabi F, Dinarvand R. "New insights into designing hybrid nanoparticles for lung cancer: Diagnosis and treatment." Journal of Controlled Release, vol. 295, pp. 250-267, Feb 2019.

[12] Akhter S, Amin S, Ahmad J, Khan S, Anwar M, Ahmad MZ, et al. "Nanotechnology to Combat Multidrug Resistance in Cancer." Resistance to Targeted ABC Transporters in Cancer, vol. 4, pp. 245-272, 2015.

[13] Tseng C-L, Wu SY-H, Wang W-H, Peng C-L, Lin F-H, Lin C-C, et al. "Targeting efficiency and biodistribution of biotinylatedEGF-conjugated gelatin nanoparticles administered via aerosol delivery in nude mice with lung cancer." Biomaterials, vol. 29, issue 20, pp. 3014-3022, Jul 2008.

[14] Elsayed I, AbouGhaly MHH. "Inhalable nanocomposite microparticles: preparation, characterization and factors affecting formulation." Expert Opinion on Drug Delivery, vol. 13, issue 2, pp. 207-222, Feb 2016.

[15] Muralidharan $P$, Malapit M, Mallory E, Hayes D, Mansour HM. "Inhalable nanoparticulate powders for respiratory delivery." Nanomedicine: Nanotechnology, Biology, and Medicine, vol. 11, issue 5, pp. 1189-1199, Jul 2015.

[16] Lee W-H, Loo C-Y, Traini D, Young PM. “Development and evaluation of paclitaxel and curcumin dry powder for inhalation lung cancer treatment." Pharmaceutics, vol. 13, issue, pp. 1-19, Jan 2021.

[17] Yang Y, Cheow WS, Hadinoto K. "Dry powder inhaler formulation of lipid-polymer hybrid nanoparticles via electrostatically-driven nanoparticle assembly onto microscale carrier particles." International Journal of Pharmaceutics, vol. 434, issue 1-2, pp. 49-58, Sep 2012.

[18] Pilcer G, Amighi K. "Formulation strategy and use of excipients in pulmonary drug delivery." International Journal of Pharmaceutics, vol. 392, issue 1-2, pp. 1-19, Jun 2010.

[19] Yang $M$, Yamamoto $H$, Kurashima $H$, Takeuchi $H$, Yokoyama $\mathrm{T}$, Tsujimoto $\mathrm{H}$, et al. "Design and evaluation of poly (DL-lactic-co-glycolic acid) nanocomposite particles containing salmon calcitonin for inhalation." European Journal of Pharmaceutical Sciences, vol. 46, issue 5, pp. 374-380, Aug 2012.

[20] Zhang J, Wu L, Chan HK, Watanabe W. "Formation, characterization, and fate of inhaled drug nanoparticles." Advanced Drug Delivery Reviews, vol. 63, issue 6, pp. 441455, May 2011.

[21] Zhou QT, Leung SSY, Tang P, Parumasivam T, Loh ZH, Chan $\mathrm{H}-\mathrm{K}$. "Inhaled formulations and pulmonary drug delivery systems for respiratory infections." Advanced Drug Delivery Reviews, vol. 85, pp. 83-99, May 2015.
[22] Chiang P-C, Hu Y, Blom JD, Thompson DC. "Evaluating the suitability of using rat models for preclinical efficacy and side effects with inhaled corticosteroids nanosuspension formulations." Nanoscale Research Letters, vol. 5, issue 6, pp. 1010-1019, Jun 2010.

[23] Onoue S, Aoki Y, Kawabata Y, Matsui T, Yamamoto K, Sato $\mathrm{H}$, et al. "Development of inhalable nanocrystalline solid dispersion of tranilast for airway inflammatory diseases." Journal of Pharmaceutical Sciences, vol. 100, issue 2, pp. 622-633, Feb 2011.

[24] Gradon L, Sosnowski TR. "Formation of particles for dry powder inhalers." Advanced Powder Technology, vol. 25, issue 1, pp. 43-55, Jan 2014.

[25] Alhajj N, O'Reilly NJ, Cathcart H. “Designing enhanced spray dried particles for inhalation: A review of the impact of excipients and processing parameters on particle properties." Powder Technology, vol. 384, pp. 313-331, May 2021.

[26] Yu S, Pu X, Ahmed MU, Heidi HY, Mutukuri TT, Li J, et al. “Sprayfreeze-dried inhalable composite microparticles containing nanoparticles of combinational drugs for potential treatment of lung infections caused by Pseudomonas aeruginosa." International Journal of Pharmaceutics, vol. 610, pp. 121160-121169, Dec 2021.

[27] Cheow WS, Ng MLL, Kho K, Hadinoto K. “Spray-freeze-drying production of thermally sensitive polymeric nanoparticle aggregates for inhaled drug delivery: effect of freeze-drying adjuvants." International Journal of Pharmaceutics, vol. 404, issue 1, pp. 289-300, Feb 2011.

[28] Wang $Y$, Kho K, Cheow WS, Hadinoto K. "A comparison between spray drying and spray freeze drying for dry powder inhaler formulation of drug-loaded lipid-polymer hybrid nanoparticles." International Journal of Pharmaceutics, vol. 424, issue 1-2, pp. 98-106, Mar 2012.

[29] Nahar K, Gupta N, Gauvin R, Absar S, Patel B, Gupta V, et al. "In vitro, in vivo and ex vivo models for studying particle deposition and drug absorption of inhaled pharmaceuticals." European Journal of Pharmaceutical Sciences, vol. 49, issue 5, pp. 805-818, Aug 2013.

[30] Mitchell JP, Nagel MW. "Cascade impactors for the size characterization of aerosols from medical inhalers: their uses and limitations." Journal of Aerosol Medicine, vol. 16, issue 4, pp. 341-377, Dec 2003.

[31] Chow MYT, Tai W, Chang RYK, Chan H-K, Kwok PCL. "In vitroin vivo correlation of cascade impactor data for orally inhaled pharmaceutical aerosols." Advanced Drug Delivery Reviews, vol. 177, pp. 113952-113961, Oct 2021.

[32] Yi D, Price A, Panoskaltsis-Mortari A, Naqwi A, Wiedmann TS. "Measurement of the distribution of aerosols among mouse lobes by fluorescent imaging." Analytical Biochemistry, vol. 403, issue 1-2, pp. 88-93, Aug 2010.

[33] Mansour HM, Rhee YS, Wu X. "Nanomedicine in pulmonary delivery." International journal of nanomedicine, vol. 4, pp. 299-319, Dec 2009.

[34] Borchard G, Cassará ML, Roemelé PEH, Florea BI, Junginger HE. "Transport and local metabolism of budesonide and fluticasone propionate in a human bronchial epithelial cell line (Calu-3)." Journal of Pharmaceutical Sciences, vol. 91, issue 6, pp. 1561-1567, Jun 2002.

[35] Selg E, Ewing P, Acevedo F, Sjöberg C-O, Ryrfeldt ^̊, Gerde $P$. “Dry powder inhalation exposures of the endotracheally intubated rat lung, ex vivo and in vivo: the pulmonary pharmacokinetics of fluticasone furoate." Journal of Aerosol Medicine and Pulmonary Drug Delivery, vol. 26, issue 4, pp. 181-189, Aug 2013.

[36] Sakagami M. "In vivo, in vitro and ex vivo models to 
assess pulmonary absorption and disposition of inhaled therapeutics for systemic delivery." Advanced Drug Delivery Reviews, vol. 58, issue 9, pp. 1030-1060, Oct. 2006.

[37] Doan TVP, Grégoire N, Lamarche I, Gobin P, Marchand S, Couet W, et al. "A preclinical pharmacokinetic modeling approach to the biopharmaceutical characterization of immediate and microsphere-based sustained release pulmonary formulations of rifampicin." European Journal of Pharmaceutical Sciences, vol. 48, issue 1, pp. 223-240, Jan 2013.

[38] RundfeldtC,SteckelH,Scherliess H,WyskaE, WlaźP.“Inhalable highly concentrated itraconazole nanosuspension for the treatment of bronchopulmonary aspergillosis." European Journal of Pharmaceutics and Biopharmaceutics, vol. 83, issue l, pp. 44-53, Jan 2013.

[39] Conde J, Tian F, Hernández Y, Bao C, Cui D, Janssen K-P, et al. "In vivo tumor targeting via nanoparticle-mediated therapeutic siRNA coupled to inflammatory response in lung cancer mouse models." Biomaterials, vol. 34, issue 31 , pp. 7744-7753, Oct 2013.

[40] Fröhlich E, Salar-Behzadi S. "Toxicological assessment of inhaled nanoparticles: role of in vivo, ex vivo, in vitro, and in silico studies." International Journal of Molecular Sciences, vol. 15, issue 3, pp. 4795-4822, Mar 2014. 